\title{
Emulsion Stability-Experimental Studies on Simultaneous Flocculation and Creaming
}

\author{
S. R. REDDY ${ }^{1}$ AND H. S. FOGLER \\ Department of Chemical Engineering, University of Michigan, Ann Arbor, Michigan 48109 \\ Received April 16, 1980; accepted October 30, 1980
}

\begin{abstract}
An experimental technique has been developed to study the emulsion stability with simultaneous flocculation and creaming. Experiments were carried out by using different ionic strengths, two different dispersants in water $\left(\mathrm{C}_{36} \mathrm{H}_{74}\right.$ and $\left.\mathrm{C}_{28} \mathrm{H}_{58}\right)$ and different particle size distributions. Cyclic changes in particle size distributions (PSD) are observed when flocculation and creaming occur simultaneously. Initially, the PSD shifts to the right toward larger particle sizes and then reverses and shifts to the left toward smaller particle sizes. This reverse shift in PSD had been observed both in theory and experiment. There are no adjustable parameters in the theory and the agreement between the theoretically predicted changes in PSD and those observed experimentally is excellent.
\end{abstract}

\section{INTRODUCTION}

The theory of emulsion stability was studied (1) by including Brownian flocculation, sedimentation flocculation, and creaming. A general dynamic equation (GDE) for simultaneous flocculation and creaming derived from a population balance was solved numerically. The solution to the general equation gives the changes in particle concentration and particle size distribution as a function of time and position in the direction of the gravitational field.

Experimental studies on emulsion stability involving simultaneous flocculation and creaming have not been reported. Emulsion flocculation has been studied (2-7) by avoiding or by neglecting creaming. Investigators in the aerosol field studied only an approximate problem involving flocculation and sedimentation of aerosols. Okuyama (8) studied the aerosol behavior in a closed chamber involving coagulation and settling. They assumed that the aerosol concentration and size distribution were spatially uniform and measured the amount of aerosol deposited at the bottom due to sedimenta-

\footnotetext{
1 Present Address: General Motors Research Laboratories, Warren, Michigan 48090.
}

tion and measured only the lumped changes in aerosol concentration and size distribution as a function of time alone. Simultaneous flocculation and creaming produces large concentration gradients in the system. The experimental studies on simultaneous flocculation and creaming require particle size distribution measurements as a function of time and position. The numerical solution to the GDE for simultaneous flocculation and creaming yielded some very interesting results that otherwise would not have been realized; namely, the cyclic change in local particle size distribution which is worth studying experimentally.

Ultrasonically formed emulsions provide an excellent opportunity for the experimental study of various interesting things that were predicted theoretically. By the ultrasonic emulsification method one can easily prepare emulsions containing a desired particle size and size distribution. Li and Fogler $(9,10)$ have shown that in ultrasonic emulsification, particle size is a function of ultrasonic irradiation time. Shorter irradiation produces larger particles and longer irradiation produces smaller particles. The stability of an emulsion can also be varied by varying the concentration of electrolyte in the water. 
In this way, one can prepare an emulsion of the desired particle size and size distribution and electrostatic stability.

\section{THEORY}

The theory of emulsion stability involving simultaneous flocculation and creaming was discussed in detail in a previous paper (11). There are three kinds of processes that take place in the emulsion. In Brownian flocculation, particles under Brownian motion collide with each other and form larger particles. In creaming, particles rise to the top due to the difference in the densities of the particles and the medium. In sedimentationflocculation, differential creaming rates of particles of different sizes cause collisons and flocculation. A general dynamic equation including all three processes was derived using the sectionalization technique with sectional incrementing based on particle radius (11):

$$
\begin{aligned}
\frac{\partial N_{k}(y, t)}{\partial t}=- & \hat{U}_{k} \frac{\partial N_{k}(y, t)}{\partial x}+\frac{1}{2} \sum_{i=1}^{k-1} \sum_{j=1}^{k-1} 1{ }^{1} \bar{\beta}_{i, j, k} N_{i}(y, t) N_{j}(y, t)-N_{k}(y, t) \sum_{i=1}^{k-1}{ }^{2} \bar{\beta}_{i, k} N_{i}(y, t) \\
& -\frac{1}{2}{ }^{3} \bar{\beta}_{k, k} N_{k}^{2}(y, t)-N_{k}(y, t) \sum_{i=k+1}^{n}{ }^{4} \bar{\beta}_{i, k} N_{i}(y, t), \quad k=1,2,3, \ldots, n, \quad[1]
\end{aligned}
$$

where

$N_{k}=$ the concentration of particles in section $k(11)$,

$\hat{U}_{k}=$ the average creaming velocity of particles in section $k(11)$, and ${ }^{1} \bar{\beta}_{i, j, k},{ }^{2} \bar{\beta}_{i, k},{ }^{3} \bar{\beta}_{k, k},{ }^{4} \bar{\beta}_{i, k}=$ inter- and intrasectional flocculation coefficients (11).

This equation was solved numerically to predict trends for various initial particle concentrations and size distributions and electrostatic stabilizations (11). The theoretical predictions will now be compared with experimental observations.

\section{EXPERIMENTAL}

Emulsions of hexatriacontane, $\mathrm{C}_{36} \mathrm{H}_{74}$, and octacosane, $\mathrm{C}_{28} \mathrm{H}_{58}$, in water were prepared ultrasonically. The acoustic emulsification technique is described in detail by $\mathrm{Li}$ and Fogler $(9,10)$. The paraffins $\mathrm{C}_{28}$ and $\mathrm{C}_{36}$ are solid at room temperature and liquid at high temperatures. Emulsions were prepared and stored at $80^{\circ} \mathrm{C}$ in the case of $\mathrm{C}_{36}$ and $65^{\circ} \mathrm{C}$ in the case of $\mathrm{C}_{28}$. The desired properties were measured at the same temperature. Whenever an emulsion sample is to be analyzed for particle size distribution, $1 \mathrm{ml}$ of emulsion is withdrawn and rapidly cooled so that the particles become solid. The particle size and size distribution are measured with the aid of a scanning electron microscope. This method is very convenient and accurate when compared to other conventional methods, such as the Coulter counter, light microscope, etc.

Experimental studies on simultaneous flocculation and creaming require the measurement of local particle size and particle size distribution as a function of time. It is not possible to withdraw samples from different depths of the system without disturbing and altering the system. Therefore, a method using two cylindrical containers, one with a narrow cross section, $\mathrm{A}$, and another with a wide cross section, B, was developed.

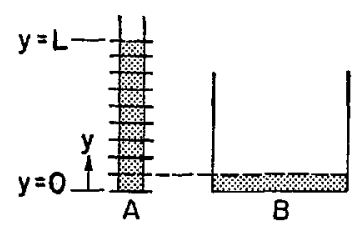

Numerical solution to the GDE requires that the emulsion system be divided axially into a number of segments of thickness, $\Delta y$. The value of $\Delta y$ is chosen such that 
computations are sufficiently accurate, yet are able to be carried out within a reasonable computing time. For these studies, the segment $\Delta y$ was chosen as $0.5 \mathrm{~cm}$, and within each segment the particle concentration and size distribution are assumed uniform. Particle concentration and size distribution in each segment are computed as a function of time. The experimental method is in accordance with the numerical technique. Partial size and size distribution are measured in a particular segment.

Container A was filled with emulsion up to the top and container B was filled up to $0.5 \mathrm{~cm}$ in height. Container $\mathrm{A}$ is divided into segments with $\Delta y=0.5 \mathrm{~cm}$. The particle concentration and size distribution in $B$ is identical to the one in the first segment of A. Particles flocculate and grow in both the first segment of $\mathrm{A}$ and in $\mathrm{B}$. Particles cream from the first segment of $A$ and enter into the second segment, whereas, in the case of $B$, particles cream and collect at the surface as a thin layer of oil. In other words, a sample from $B$ represents the emulsion in the first segment of A. Samples from the top of container A and from B were drawn for particle analysis. In this way we were able to measure the particle size and size distribution at $y=0$ and $y=L$. Container B was chosen to be a wider cross section for convenience in taking out the samples and also samples can be drawn without altering the level. However, no effect of container size was noticed when tried with different size containers.

Paraffin oil-in-water emulsions were prepared ultrasonically by using a model W-185E Branson Sonic Power Company Sonifier. Sixty milliliters of an emulsion containing $0.05 \mathrm{ml}$ of $\mathrm{C}_{36}$ or $\mathrm{C}_{28}$ was prepared by a few minutes of irradiation. The irradiation time is chosen so that the resulting emulsion will contain a particle size on the order of $1 \mu \mathrm{m}$. Electrostatic stability of the ultrasonic emulsion is adjusted by adding magnesium sulfate. Irradiation time and concentration of $\mathrm{MgSO}_{4}$ are chosen such that flocculation and creaming will take place at a significant rate. Immediately after irradiation, the emulsion was transferred into preheated containers A and B. Containers A and B were covered with aluminum foil, and were maintained at a constant temperature of $80^{\circ} \mathrm{C}$ in a preheated electric oven. They were left in the oven for several hours without being disturbed. Samples were drawn from the top of $A$ and $B$ in order to measure the particle size and distribution by using a scanning electron microscope (9).

One of the primary goals of the experimental study was to look for the theoretically predicted cyclic changes in particle size distribution in an emulsion where flocculation and creaming are taking place simultaneously. After a few preliminary experiments, it was possible to arrive at suitable particle concentration, size distribution, and electrostatic stability for the study of simultaneous flocculation and creaming. The desired emulsion properties required were such that the flocculation and creaming will occur at a significant rate.

\section{RESULTS AND DISCUSSION}

The particle size and size distributions were measured by taking samples from container $\mathrm{A}$ and container $\mathrm{B}$ at various times during the experiment. The initial particle size and size distribution are the same at the bottom, $y=0$, and top of the column, $y=L$. Three experiments with different initial particle size distributions and different electrostatic properties are presented. The initial particle concentration, size distribution, and electrostatic properties of the emulsion were used to predict the particle size distribution as a function of time and position by solving the GDE numerically (11). The experimentally measured particle size distributions were then compared with theoretically predicted results.

The changes in particle size and size distribution in the emulsion can be observed from the electron photomicrographs. From Fig. 1, one observes that the average par- 

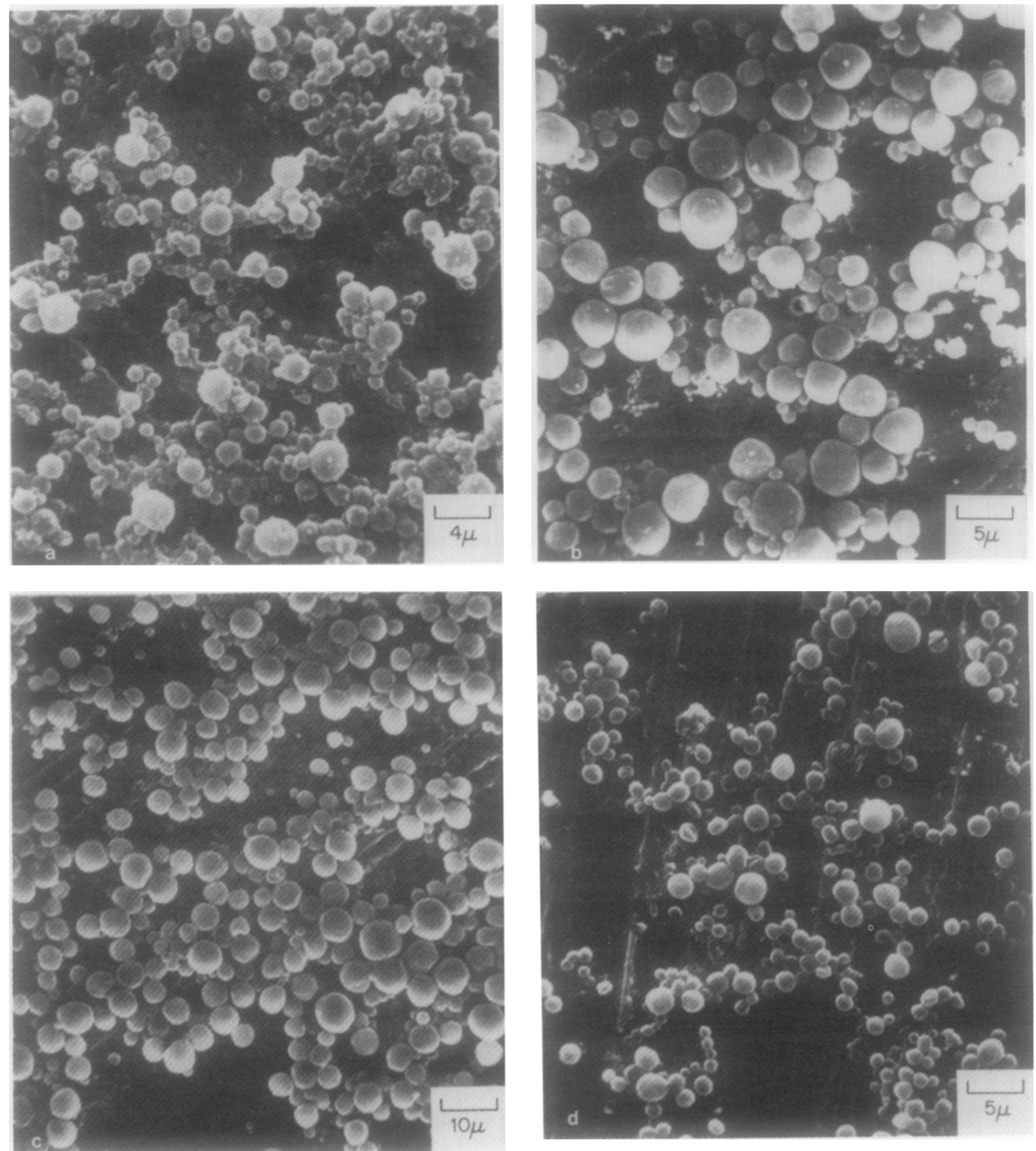

FIG. 1. Scanning electron photomicrographs showing the changes in particle size as a function of time and position. (a) $t=0 ; 0<y<L$. (b) $t=1 \mathrm{hr} ; 0<y<L$. (c) $t=4 \mathrm{hr} ; y=L$. (d) $t=4 \mathrm{hr} ; y=0$.

ticle size at the point corresponding to the bottom of the vessel, $y=0$, increases initially and then decreases. In other words, initially, smaller particles coagulate and form relatively stable larger particles (photomicrograph 1b). The larger particles then cream out leaving smaller particles behind (photo- micrographs $1 \mathrm{c}$ and $\mathrm{d})$. The particle size and size distribution are functions of position; notice that particles at $y=\mathbf{0}$ (photomicrograph 1d) are much smaller than those which are at the top, $y=L$ (photomicrograph 1c). The average particle size increases and then decreases at the lower end of the column 
and particle size is a strong function of position.

Particles of different sizes were counted and classified into groups based on particle diameter. Particle size distributions were calculated for all samples. In the following figures, particle size distributions are plotted as number density as a function of particle diameter (microns). In other words,

Number density $=P(D) / N$,

$(P(D) d D) / N$

$=$ the fraction of all the particles with diameters between $D$ and $D+d D$,

where

$P(D)=$ the particle size distribution function

$N=$ the total number of particles.

The area under the curve is equal to unity, i.e.,

$$
\int_{0}^{\infty} \frac{P(D)}{N} d D=1 .
$$

The particle size distributions (PSD) at various aging times corresponding to $y=0$ (i.e., the PSD in the first segment is represented as PSD at $y=0$ ) are plotted in Fig. 2. PSDs computed from the theory of simultaneous flocculation and creaming are also shown in this figure for the electrical and

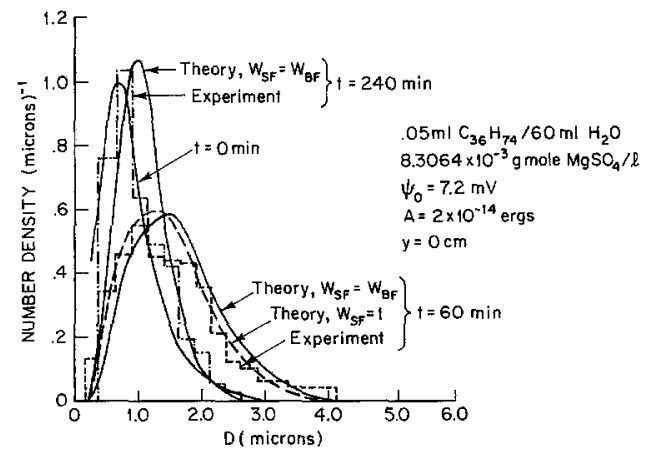

FIG. 2. Changes in the particle size distribution with time at $y=0$ for the $\mathrm{C}_{36} \mathrm{H}_{74}$ /water emulsion containing $8.3064 \times 10^{-3} \mathrm{~g}$ mole/liter $\mathrm{MgSO}_{4}$.

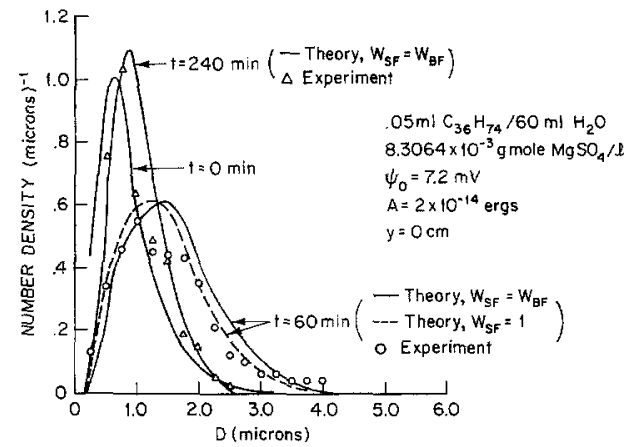

FIG. 3. Simplified form of Fig. 2.

physical properties specified in the appendix. The initial PSD and electrostatic properties of the emulsion were used for the theoretical predictions. Figure 2 can be elucidated by presenting the experimental data as midpoints on each histogram instead of presenting the complete set of histograms as shown in Fig. 3. From this figure, one observes the particle size distribution initially shifts to the right toward the larger particle sizes and then to the left toward the smaller particle sizes. This experimentally observed reverse shift in the size distribution is what was theoretically predicted. Initially, the rate of flocculation is significant until about $t=60 \mathrm{~min}$ and then the rate of creaming becomes significant. Flocculation of particles produces the shift in size distribution to the right and creaming produces the shift in size distribution to the left. The cyclic changes in

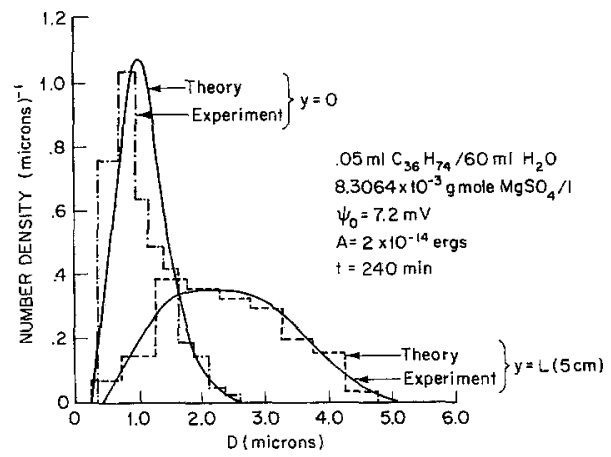

FIG. 4. Particle size distribution at $y=0$ and $y=L$ $\left(5 \mathrm{~cm}\right.$ ) after $4 \mathrm{hr}$ for the $\mathrm{C}_{36} \mathrm{H}_{74} /$ water emulsion containing $8.3064 \times 10^{-3} \mathrm{~g}$ mole/liter $\mathrm{MgSO}_{4}$. 


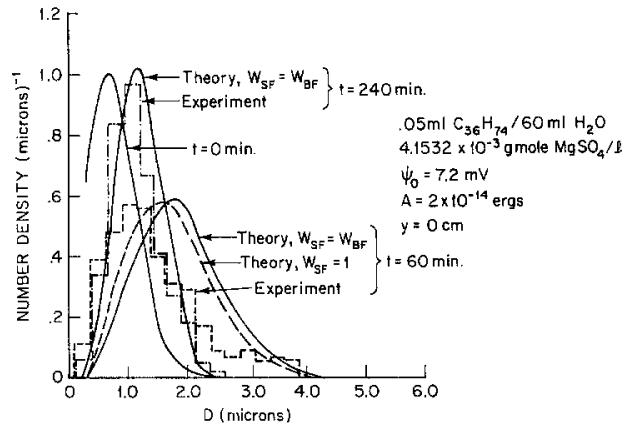

FIG. 5. Changes in the particle size distribution with time at $y=0$ for the $\mathrm{C}_{36} \mathrm{H}_{74} /$ water emulsion containing $4.1532 \times 10^{-3}$ g mole/liter $\mathrm{MgSO}_{4}$

emulsion particle size distribution are due to simultaneous flocculation and creaming. One observes that the agreement between the theory and experiment is good.

The emulsion particle size distribution is a strong function of position in the gravitational field. The particle size distribution at $t=240 \mathrm{~min}$ corresponding to two different locations, $y=0$ and $y=L$, are presented in Fig. 4 along with the theoretically predicted size distributions. In the bottom segment, $y=0$, the larger particles are leaving, as a result of creaming, thereby causing an increase in the relative concentration of smaller particles resulting in a narrow particle size distribution. Larger particles are entering the top segment producing an increase in the concentration of larger particles at the top segment, $y=L$. The net result is a smaller

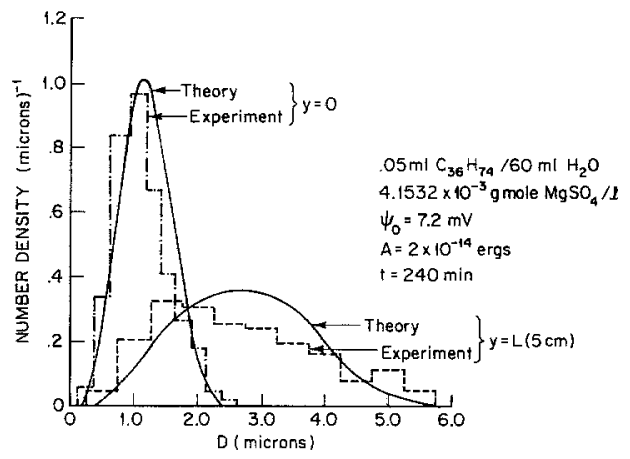

FIG. 6. Particle size distribution at $y=0$ and $y=L$ $(5 \mathrm{~cm})$ after $4 \mathrm{hr}$ for the $\mathrm{C}_{36} \mathrm{H}_{74} /$ water emulsion containing $4.1532 \times 10^{-3} \mathrm{~g}$ mole/liter $\mathrm{MgSO}_{4}$.

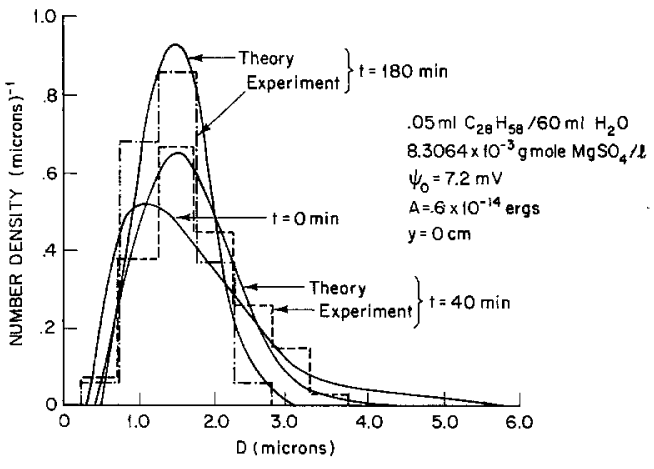

FIG. 7. Changes in the particle size distribution with time at $y=0$ for the $\mathrm{C}_{28} \mathrm{H}_{58}$ /water emulsion containing $8.3064 \times 10^{-3} \mathrm{~g}$ mole/liter $\mathrm{MgSO}_{4}$.

particle size and narrower distribution in the lower part, and larger and wider particle size distribution in the upper part of the column. Again, agreement between the theory and experiment is quite good.

The experimental studies had been carried out by using different systems. The $\mathrm{C}_{36} \mathrm{H}_{74}$ in water emulsion with different initial particle size distributions and ionic strengths had been used and the results are shown in Figs. 5 and 6 . The corresponding particle size distributions were also predicted from the theory and are also presented in Figs. 5 and 6 . It should be noted here that there is no adjustable parameter in the theory. The same Hamaker constant and surface potential was used for the $\mathrm{C}_{36} \mathrm{H}_{74}$ analysis. In another experiment, $\mathrm{C}_{28} \mathrm{H}_{58}$ was emulsified

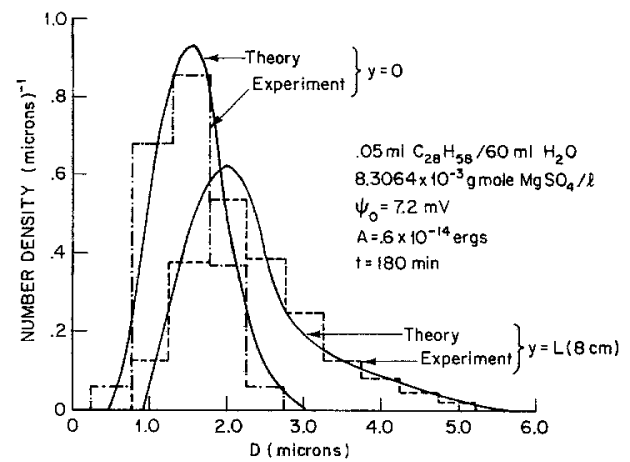

FIG. 8. Particle size distribution at $y=0$ and $y=L$ $\left(8 \mathrm{~cm}\right.$ ) after $3 \mathrm{hr}$ for the $\mathrm{C}_{28} \mathrm{H}_{58} /$ water emulsion containing $8.3064 \times 10^{-3} \mathrm{~g}$ mole/liter $\mathrm{MgSO}_{4}$. 


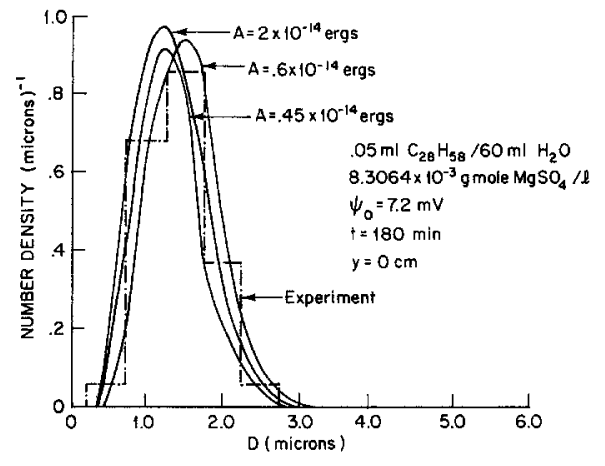

FIG. 9. Comparison of theory and experiment for various values of the Hamaker constant.

in water and the results of these experiments are shown in Figs. 7 and 8. In this case, the initial particles are very large, hence flocculation is not very significant, and the PSD will not shift far to the right toward the larger particle size. The PSD reverses and becomes narrow with time and PSD is a function of position, as shown in Fig. 8.

The theoretical distributions were calculated using two different values of the sedimentation flocculation stability factor $\left(W_{S F}\right)$ in Figs. $2,3,4,5$, and 6. In one case, $W_{\mathrm{SF}}$ was taken to be equal to the Brownian flocculation stability factor $\left(\mathrm{W}_{\mathrm{BF}}\right)$, which is easily calculated from DLVO theory (11). In the other case, $\mathrm{W}_{\mathrm{SF}}$ was set equal to one, which is equivalent to no electrostatic barrier for sedimentation flocculation. Both cases yield similar but not identical results.
A value for the surface potential, $\Psi=7.2$ $\mathrm{mV}$, was used for all theoretical predictions. This value was obtained from studies on the stability of acoustic emulsions (13). Theoretically predicted particle size distributions are very sensitive to the Hamaker constant, $A$. Experimental results were compared with theoretical predictions by using various values of the Hamaker constant and the results are presented in Fig. 9. Literature values of $A$ vary over two orders of magnitude, $10^{-15}$ to $10^{-13} \mathrm{erg}$, and differ for $\mathrm{C}_{28} \mathrm{H}_{58}$ and $\mathrm{C}_{36} \mathrm{H}_{74}$ (12). It is clear from Fig. 9 that the experimental and theoretical particle size distributions agree only for a particular value of $A$.

\section{SUMMARY}

An experimental technique was developed to study emulsion stability involving simultaneous flocculation and creaming. Suitable emulsions for the study were prepared by emulsifying $\mathrm{C}_{36} \mathrm{H}_{74}$ or $\mathrm{C}_{28} \mathrm{H}_{58}$ in water ultrasonically. Electrostatic stability to flocculation was adjusted by varying the ionic strength. Changes in particle size and size distribution were measured as a function of time and position. The changes in particle size and size distribution fit very well to the theoretically predicted distributions. The reverse shift in particle size distribution was observed in both theory and experiment.

APPENDIX

\begin{tabular}{llll}
\hline \multicolumn{1}{c}{ Parameter } & \multicolumn{1}{c}{ Figs. 2, 3, and 4} & \multicolumn{1}{c}{ Figs. 5 and 6 } & \multicolumn{1}{c}{ Figs. 7, 8, and 9 } \\
\hline Paraffin & $\mathrm{C}_{36} \mathrm{H}_{74}$ & $\mathrm{C}_{36} \mathrm{H}_{74}$ & $\mathrm{C}_{28} \mathrm{H}_{58}$ \\
Temperature & $80^{\circ} \mathrm{C}$ & $80^{\circ} \mathrm{C}$ & $65^{\circ} \mathrm{C}$ \\
Density of continuous medium & $0.97 \mathrm{~g} / \mathrm{cm}^{3}$ & $0.97 \mathrm{~g} / \mathrm{cm}^{3}$ & $0.99 \mathrm{~g} / \mathrm{cm}^{3}$ \\
Density of dispersant & $0.75 \mathrm{~g} / \mathrm{cm}^{3}$ & $0.75 \mathrm{~g} / \mathrm{cm}^{3}$ & $0.733 \mathrm{~g} / \mathrm{cm}^{3}$ \\
Viscosity of continuous medium & $0.37 \mathrm{cP}$ & $0.37 \mathrm{cP}$ & $0.44 \mathrm{cP}$ \\
Initial particle concentration & $6.058 \times 10^{8} \mathrm{part} / \mathrm{cm}^{3}$ & $1.698 \times 10^{9} \mathrm{part} / \mathrm{cm}^{3}$ & $1.3234 \times 10^{8} \mathrm{part} / \mathrm{cm}^{3}$ \\
2-2 Electrolyte concentration & & & \\
$\quad$ (MgSO & & \\
Dielectric constant of continuous & $8.3064 \mathrm{~g} \mathrm{~mole} / \mathrm{liter}$ & $4.1532 \mathrm{~g} \mathrm{~mole} / \mathrm{liter}$ & $8.3064 \mathrm{~g} \mathrm{mole/liter}$ \\
$\quad$ & & & \\
medium & 61.00 & 61.00 & 65.00 \\
Surface potential (1) & $7.2 \mathrm{mV}$ & $7.2 \mathrm{mV}$ & $7.2 \mathrm{mV}$ \\
Hamaker constant & $2 \times 10^{-14} \mathrm{erg}$ & $2 \times 10^{-14} \mathrm{erg}$ & $0.45 \times 10^{-14} \mathrm{erg}$ \\
Wavelength (11) & $10^{-5} \mathrm{~cm}$ & $10^{-5} \mathrm{~cm}$ & $10^{-5} \mathrm{~cm}$ \\
\hline
\end{tabular}




\section{REFERENCES}

1. Reddy, S. R., Ph.D. Thesis, University of Michigan, 1981.

2. Fitch, R. M., and Watson, R. C., J. Colloid Interface Sci. 68, 14 (1979).

3. Suwa, T., Watanabe, T., Okamoto, J., and Machi, S., Lobunshi Renbunshu 35, 236 (1978).

4. Bernstein, D. B., Ph.D. Thesis. University of Michigan, 1971.

5. Sherman, P. (Ed.), "Emulsion Science." Academic Press, New York, 1968.

6. Smith, A. L. (Ed.), "Theory and Practice of Emulsion Technology." Section 4. Academic Press, New York, 1976.
7. Kako, M., and Kondo, S, J. Colloid Interface Sci. 69, 163 (1979).

8. Okuyama, K., Kousaka, Y., and Yoshida, T., J. Chem. Eng. Japan 9, 140 (1976).

9. Li, M. K., and Fogler, H. S., J. Fluid Mech. 88, 499 (1978).

10. Li, M. K., and Fogler, H. S., J. Fluid Mech. 88, 513 (1978).

11. Reddy, S. R., Melik, D. H., and Fogler, H. S., "Emulsion Stability--Theoretical Studies on Simultaneous Flocculation and Creaming," Journal of Colloid and Interface Science 82, 116, (1981).

12. Lyklema, J., Advan. Colloid Interface Sci. 2, 65 (1968).

13. Reddy, S. R., and Fogler, H. S., J. Phys. Chem. 84, 1570 (1980). 